\title{
Search and Rescue Optimization Algorithm - Second Order Sliding Mode Control: AUV Error Tracking
}

\author{
Rupam Gupta Roy \\ Department of Electronics and Instrumentation Engineering \\ National Institute of Technology \\ Agartala, Tripura, India \\ royrupamgupta@gmail.com
}

\author{
Dibyendu Ghoshal \\ Department of Electronics and Communication Engineering \\ National Institute of Technology \\ Agartala, Tripura, India
}

\begin{abstract}
Controlling, directing, and navigating AUVs are challenging and considered complicated, and it is evaluated to the performance of the autonomous surface-level. The motion can be controlled in such vehicles, based upon the evaluation of indefinite hydrodynamic forces and the disturbances which happen in this vehicle in the background of underwater. In this work, the Search and Rescue Optimization (SRO) algorithm is done in AUV beside the SR-SoSMC to control the yaw angle. The most important principle of the work is to minimize error which happens in the system among the desired signal and a controlled signal in proportion to yaw angle. Exploiting this proposed technique, both the incidence of performance of the controller and chattering of the AUV system can be reduced. In addition, the proposed algorithm is evaluated with conventional algorithms. From the experimental analysis, it reveals that the proposed algorithm performance regarding error analysis is improved when compared with the conventional algorithms such as Genetic Algorithm (GA)-SoSMC, Firefly (FF)-SoSMC, Grey Wolf Optimization (GWO)-SMC, Artificial Bee Colony (ABC)- SoSMC and Group Search Optimization (GSO)-SoSMC.
\end{abstract}

Keywords: AUV; Sliding Mode Controller; Yaw Angle; Error; Optimization Algorithm

\begin{tabular}{ll} 
Nomenclature \\
\hline Abbreviations & Descriptions \\
\hline SMC & Sliding Mode Control \\
ESO & Extended State Observer \\
SNs & Sensor Nodes \\
AUV & Autonomous Underwater Vehicle \\
TOC & Time-Optimal Control \\
PDSR & Packet Delivery Success Rate \\
IBPDR & Intermittent Belief Propagation Based Dead Reckoning \\
UABP & Underwater-Adaptive Belief Propagation \\
MU & Maximum Unsuccessful \\
PFC & Predictive Functional Control \\
ADRC & Active Disturbance Rejection Control \\
MC-RESO & Model-Compensation RESO \\
GA & Genetic Algorithm \\
NN & Neural Network \\
SoSMC & Second order Sliding Mode Control approach \\
SE & Social Effect \\
RESO & Reduced-Order ESO \\
SE & Social Effect \\
CB & Center of Buoyancy \\
PTOC & Proximate Time-Optimal Control \\
CL & Cooperative Localization \\
MC-ESO & Model-Compensation ESO \\
FoSMC & First order-SMC \\
USN & Unsuccessul Search Number \\
GBNN & Glasius Bio-inspired Neural Network \\
WPT & Wireless Power Transfer \\
HOSMC & High Order Sliding Mode Control \\
\hline OResbe Publishers
\end{tabular}

(C)Resbee Publishers

https://doi.org/10.46253/jcmps.v3i1.a2 


\section{Introduction}

In recent research, AUV is considered a significant model; however, one particular AUV cannot determine the sophisticated, broad area in the detection of coverage tasks owing to its reduced energy [1]. Then, concerted multi-AUVs complete coverage mechanism enters into that processes the numerous robots set up a coordinated strategy to determine the full analysis of defined regions completely through a particular collaborative method. It requires the integration of path planning and multi-vehicle service. So, there were few investigations in the method for complete coverage path planning of multi-robot cooperative [3].

The AUV undergoes multi-axis complex nonlinear motion paths in existence of external disturbances, like tides, waves, currents, and a downward or upward stream in an underwater mission. In addition, its dynamics are varying considerably with the alteration in extraneous operating circumstances, leads to the absence of knowledge regarding coefficients of hydrodynamic. In several undersea conditions, aforesaid challenges are precisely evaluated [5].

Nevertheless, a huge number of algorithms are present to design the coefficients of hydrodynamic that are usually inappropriateness [15] [18]. These types of complications generally take regarding significant designing with lack of accuracy for dynamics of AUVs and thus the system existence concerns are yet large [6]. Owing to unsymmetrical axes of AUV, one more new issue is nonlinear effective crosscoupling effects. Finally, the implements of cross-coupling become certainly outstanding in actual AUV strategy operating in uncertain natural circumstances. To confront these obstacles, numerous control approaches were implemented during the past decade, to the underwater vehicles' motion control, by investigators. But, there yet exists a tendency to attain firmness in existence of inauspicious undersea environmental circumstance and uncertainties of parametric.

Hence, for undersea vehicles, high relevant control strategies have been applied in conventional research. Among them are SMC [8], linear PD/PID control [9], self-tuning control [10], adaptive control [12], output feedback control [13], gaussian control of linear quadratic [15], reducedorder Hœ control [16] fuzzy logic control [17], artificial NN control [38], nonlinear control [11], GA-based control [19], gain scheduling control [14], and neuro-fuzzy control [20].

In underwater vehicle control, SMC is a potent approach constantly employed. This approach requires finite-time convergence and stability over bounded extraneous disturbances. Here, the primary execution was done, and the aforesaid controllers possess the behavior of dynamic control input owing to the signum function that leads to the undesirable chattering effect [29]. Nevertheless, there subsist numerous states to reduce the deployment in chattering manner, such as restoring the signum model using a hyperbolic tangent, saturation or sigmoid models [21] that alleviate the control signal; however, at the price of robustness loss due to the constricts of the sliding systems path to the vicinity of the sliding surface's [22]. HOSMC is considered as an additional traditional system to lessen the chattering amplitude. At last, dynamical gains [23] or SMC with auto-adjustable [24] is another solution to reduce the effect of the first order SMC disadvantages. Aforesaid approaches, an adaptive rule is recommended with the impact of disturbances to adapt the feedback controller increases corresponding. There are numerous studies adopting this reason. For example, an adaptive first-order SMC of an AUV is designed in [25] for the stabilization of the set-point. Here, the control signal was subdivided into three phrases such as initially, the term of equal control for underestimating the recognized system parameters. Next, the disturbance impact is reduced by the discontinuous signum function. In [26], for the trajectory tracking, the adaptive SMC of the pitch and yaw dynamics was developed. In a study of the controller, it is received to detail of the unknown disturbances and the dead-zones of the actuator's nonsymmetric. To adjust the controller's gains, the law disturbance, and the adaptive observer, was modeled.

The main contribution of this work is to present a controller in order to control the yaw angle of the AUV system by exploiting the SR-SoSMC algorithm. Moreover, the SRO algorithm is exploited to optimize the weight factor to minimize the tracking error. Additionally, it concentrates to reduce tracking error which happens in the AUV system. Finally, the effectiveness of the proposed SR-SoSMC algorithm is shown by evaluating several conventional algorithms.

\section{Literature Review}

In 2018, Zhengchao Yan et al [1], developed a rotation-free WPT system on the basis of a novel coil structure. It was exploited for charging autonomous underwater vehicles to attain the power of stable output and effectual over misalignments in a rotational manner. The new coil structure includes two decoupled receivers set up of two reversely wound coils of the receiver and the magnetic flux directions of the two receivers were perpendicular, assuring an approximately constant total mutual inductance. The developed coil model was corroborated by means of finite element analysis. A rotation-free was 
compensated with the LCC-LCC in correspondence with the prototype of WPT was established and the investigational outcomes find out the analytical evaluation and experimentations.

In 2018, Bing Sun et al [2] developed a novel scheme with the GBNN approach with centralized and discrete programming. Initially, fundamental designing for multi-AUVs entire coverage issues on the basis of the NN and grid map was explained. Subsequently, the model for solitary AUV entire coverage was developed on the basis of the GBNN algorithm that was a novel adopted tool with few numbers of computations and maximum effectiveness. The multi-AUV entire coverage centralized and discrete programming was developed on the basis of the GBNN algorithm to resolve the complexity.

In 2017, Bingbing Zhang et al [3], developed a novel energy-effectual tracking system to situate itself in time using UWSNs for an AUV. Initially, a tracking protocol taking into consideration the energy utilization in both the SNs and AUV was designed. As the protocol for tracking would inexorably have an effect on the packet delivery among the AUV and the SNs. The PDSR was evaluated to discard light on the parameters of the system impact for the performance of the tracking.

In 2019, Masanao Shinohara et al [4], established a measurement of the underwater gravity system for an AUV that was appropriate in broad region studies related to the seafloor with an immense solution. A developed gravimeter was grown on a leveling system and the platform was comprised in a pressure vessel. In a huge AUV, the gravimeter system was deployed. From the AUV, all the power was transferred and an acoustic transmission system facilitates monitoring and control during an investigation. The AUV was operated at a constant depth and speed in a similar profile.

In 2019, Yichen Li et al [5], developed IBPDR as a CL model. The discontinuous IBPDR feature decreases the costs of communication between AUVs by minimizing the frequency of CL. In the IBPDR model, a particle-based UABP algorithm for CL was designed. The UABP algorithm was obviously distributed and possible in non-Gaussian and nonlinear circumstances. Hence, it was appropriate for CL problems. Moreover, the UABP algorithm was forceful to the mounted up errors of the inertial measurement and minimizes costs of the communication between AUVs.

In 2018, Wilman Alonso Pineda Muñoz et al [6], focused on the PFC that was a control method that was simple to recognize, tune, install, and optimizing. PFC was found and implemented in industrial functions, like distillation, reactors, and furnaces. Moreover, it suggests the first application of the PFC in AUV, and the experimentation outcomes of PFC, gain scheduling controllers and fuzzy. By experimentations and navigation investigations at sea that profitably justify the PFC performance scheme in AUV motion control, PFC operation was analyzed with alternate control approaches like gain and fuzzy scheduling control.

In 2017, An Li, Li Ye et al [7], developed a soft-switching PTOC on the basis of the modelcompensation ESO and the fuzzy switching of Takagi-Sugeno, for the underactuated AUV. Initially, a TOC rule was determined for the underactuated AUV first-order Nomoto design on the basis of the Pontryagin's utmost rule. Subsequently, a design-compensation ADRC was proposed; the nonlinear heading dynamic model was compensated by the dominant feature to a first-order Nomoto design. The common ESO was reinstated with a RESO to minimize complications, and MC-RESO, which was modeled by including the recognized limited design to RESO.

\section{UAV Kinematics Modeling}

\subsection{Motions Equations}

As in [27], a slender body AUV in a dynamic form comprises 2 frames such as body and the inertiareferenced frame. Additionally, it is exploited to demonstrate an AUV motion. Fig. 1 states that at the CB the AUV is centered, therefore it possesses $\mathrm{x}$-axis forward as body-referenced, $\mathrm{z}$-axis to the port and $\mathrm{y}$-axis to up. Additionally, in the positive counterclockwise direction the roll $(\varphi)$ regarding $\mathrm{x}$-axis is positioned, and in positive turning towards the left, the yaw $(\psi)$ regarding the $\mathrm{y}-$ axis is positioned, and towards bow-up direction, pitch $(\theta)$ regarding the $\mathrm{z}$-axis is positive from the bridge of the body. In general, for the roll, horizontal and vertical plane; the three-dimensional model is separated into 3 subsystems in an interacting way. Moreover, the axis such as the body and inertia frames is linked regarding the rotations in relation to the yaw angle $\psi$ in $y$-axis. Hence, as in Eq. (1), the sideslip angle can be indicated, whereas $\mathrm{v}_{\mathrm{x}}$ indicates the body axis frame and likewise $\mathrm{v}_{\mathrm{y}}$ and $\mathrm{v}_{\mathrm{z}}$ indicates the inertial axis frames.

$$
\beta=\arctan \left(\frac{\mathrm{v}_{\mathrm{z}}}{\sqrt{\mathrm{v}_{\mathrm{x}}^{2}+\mathrm{v}_{\mathrm{y}}^{2}}}\right)
$$




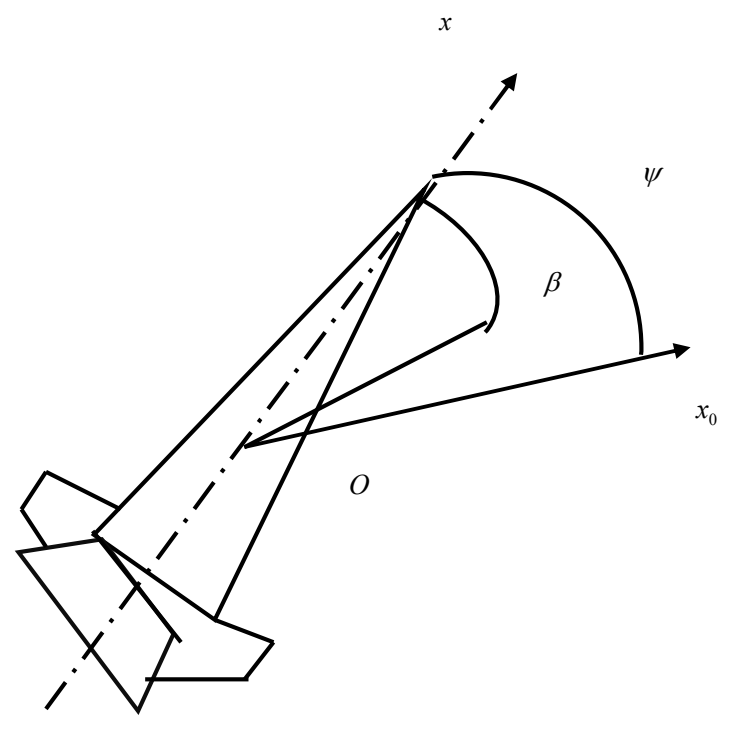

(a)

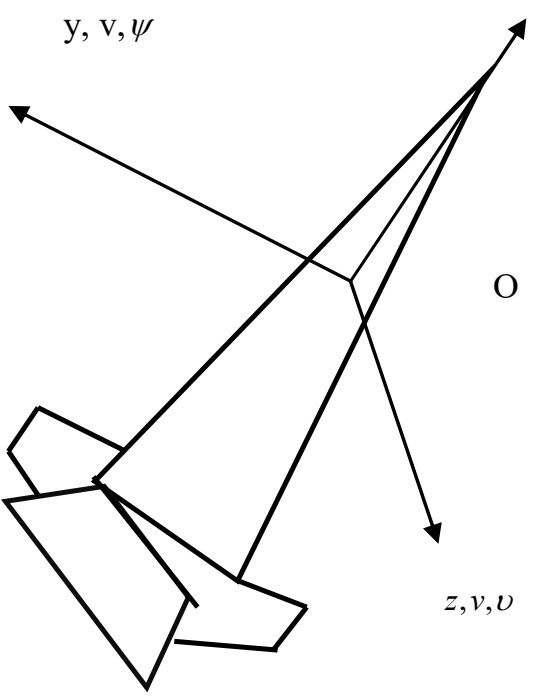

(b)

Fig. 1. An AUV body referenced coordinate system

Likewise, as in eq. (2), the angle of attack can also be indicated regarding both inertia and body axis frames. As in eq. (3) and (4), the AUV kinematics is indicated regarding the co-ordinate frame transition, whereas $\omega_{\mathrm{x}}, \omega_{\mathrm{y}}$ and $\omega_{\mathrm{z}}$ indicates as angular velocities in the body-referenced frame. Moreover, AUV integrates both parameters of hydrodynamic and nonlinear, therefore creates it multifaceted for the accomplishment of the control algorithm exploiting the linear algorithm.

$$
\begin{aligned}
& \alpha=-\arctan \left(\frac{\mathrm{v}_{\mathrm{y}}}{\mathrm{v}_{\mathrm{x}}}\right) \\
& {\left[\begin{array}{c}
\mathrm{x} \\
\mathrm{y} \\
\mathrm{z}
\end{array}\right]=\left[\begin{array}{ccc}
\cos \theta \cos \psi & \sin \psi \sin \varphi-\sin \theta \cos \psi \cos \varphi & \sin \psi \cos \varphi+\sin \theta \cos \psi \sin \varphi \\
\sin \theta & \cos \theta \cos \varphi & -\cos \theta \sin \varphi \\
-\cos \theta \sin \varphi & \cos \psi \sin \phi+\sin \theta \sin \psi \cos \varphi & \cos \psi \cos \varphi-\sin \theta \sin \psi \sin \varphi
\end{array}\right]\left[\begin{array}{c}
\mathrm{v}_{\mathrm{x}} \\
\mathrm{v}_{\mathrm{y}} \\
\mathrm{v}_{\mathrm{z}}
\end{array}\right]} \\
& {\left[\begin{array}{c}
\dot{\psi} \\
\dot{\theta} \\
\dot{\varphi}
\end{array}\right]=\left[\begin{array}{ccc}
0 & \sec \theta \cos \varphi & -\sec \theta \sin \varphi \\
0 & \sin \varphi & \cos \varphi \\
1 & -\tan \theta \cos \varphi & \tan \theta \sin \varphi
\end{array}\right]\left[\begin{array}{c}
\mathrm{w}_{\mathrm{x}} \\
\mathrm{w}_{\mathrm{y}} \\
\mathrm{w}_{\mathrm{z}}
\end{array}\right]}
\end{aligned}
$$

\subsection{Heading Dynamics}

Usually, the vehicle's control inputs comprise the differential rudder $\delta_{\mathrm{d}}$, trust $\mathrm{T}$, the elevator rudder $\delta_{\mathrm{e}}$, and the steering rudder $\delta_{\mathrm{r}}$. Moreover, T represents a trust which is indicated as a constant, and therefore the trajectory of the vehicle, is controlled using adaptable the vehicle attitude via rudders regarding the task done by the slender body AUV [27]. In the AUV attitude control with yaw and pitch angle, exploiting motion equations, the attitude dynamics are indicated as a second-order system with indefinite hydrodynamics and non-linear substance. Moreover, the pitch dynamics, roll, and yaw possess a similar pattern. Additionally, for implementation, heading control can be represented by the attitude control algorithm.

The vehicles roll is abandoned, as a result of the vehicles; a diminutive pitch angle can be implicit as revealed in Fig. 2, which is espoused from [29]. Let us the nonlinear and the disturbance substances, the kinematics formulation, and yaw dynamics, can be indicated as stated in eq. (5) and (6), whereas $C_{1}$ and $\mathrm{C}_{2}$ indicates parameters that are unidentified which are based upon the hydrodynamics coefficients, $\mathrm{C}_{3}$ indicates the control gain in the open-loop system, $\mathrm{f}\left(\mathrm{v}, \psi, \omega_{\mathrm{y}}, \mathrm{t}\right)$ indicates parameters of a nonlinear substance by means of pitch and additional motion, $\delta_{\mathrm{r}}$ represents the steering rudder control input and $d$ represents the unrevealed disturbance which happens in the vehicle. 


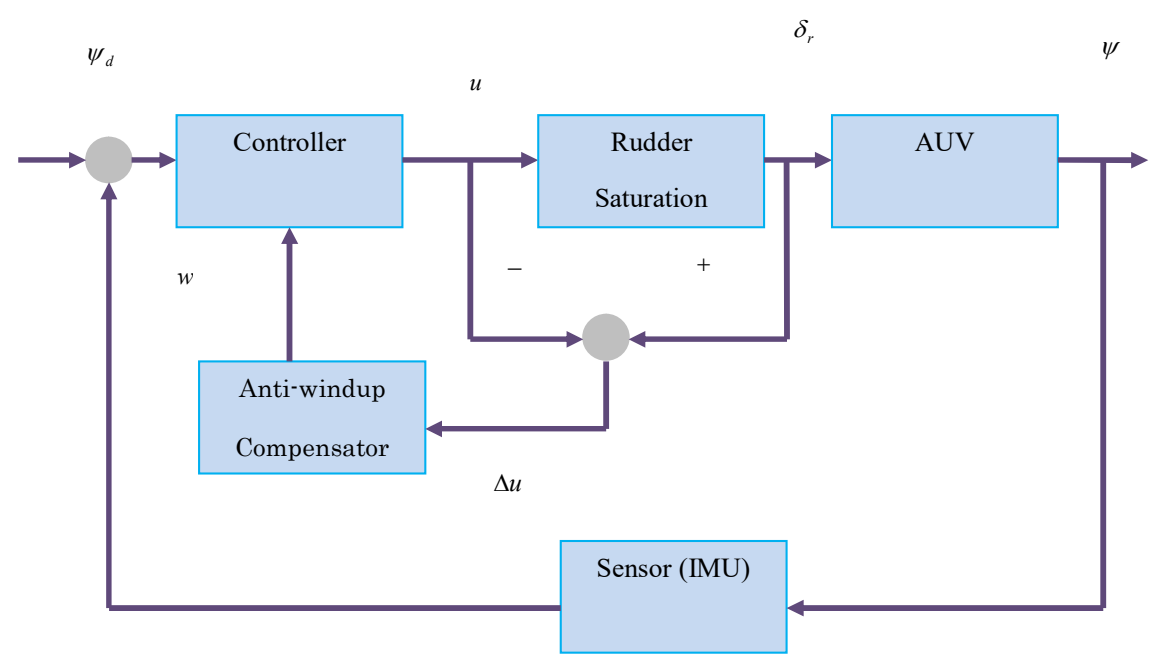

Fig. 2. System model of auxiliary compensation

$$
\psi=\omega_{\mathrm{y}}
$$

Hence, the algorithm is indicated as an easy form which is stated in eq. (7) and (8), whereas $\mathrm{h}=\left[\mathrm{C}_{1} \cdot \mathrm{C}_{2}\right]^{\mathrm{T}}, \phi=\left[\omega_{\mathrm{y}} \cdot \beta\right]^{\mathrm{T}}, \quad D$ indicates disturbance term and $\sigma$ indicates the left and right slopes. The major purpose is to control the rudder, $\psi$ regarding the desired trajectory, $\delta_{\mathrm{r}}$ to manage the yaw angle and $\psi_{\mathrm{d}}(\mathrm{t})$ indicates the infinite time interval.

$$
\begin{aligned}
& \dot{\omega}_{\mathrm{y}}=\mathrm{C}_{1} \omega_{\mathrm{y}}+\mathrm{C}_{2} \beta+\mathrm{C}_{3} \delta_{\mathrm{r}}+\mathrm{f}\left(\mathrm{v}, \psi, \omega_{\mathrm{y}}, \mathrm{t}\right)+\mathrm{d}(\mathrm{t}) \\
& \dot{\psi}=\omega_{\mathrm{y}} \\
& \dot{\omega}_{\mathrm{y}}=\mathrm{h}^{\mathrm{T}} \phi+\mathrm{C}_{3} \mathrm{mU}_{\mathrm{eq}}+\mathrm{C}_{3} \sigma+\mathrm{D}
\end{aligned}
$$

Hence, the tracking error is defined as follows:

$$
\mathrm{e}=\psi(\mathrm{t})-\psi_{\mathrm{d}}(\mathrm{t}) \rightarrow 0, \quad \mathrm{t} \rightarrow \infty
$$

As stated in eq. (10), the tracking error time derivative regarding the desired trajectory angle.

$$
\dot{\mathrm{e}}=\psi-\psi_{\mathrm{d}}=\omega_{\mathrm{y}}-\psi_{\mathrm{d}}
$$

To aid the control design; 2 statement is done for instance,

Statement 1: $\psi$ and $\dot{\psi}$ specifies the calculable outputs. Moreover, the time-deviating disturbance phrase $D$ is stated within the range $\rho \| \leq D$, whereas $\rho$ indicates the value of the unidentified upper bound which is higher than zero.

Statement 2: Control gain $\mathrm{C}_{3}$ is indefinite, however, it's upper bound and the sign is recognized $\left(0<\mathrm{C}_{3}<\overline{\mathrm{C}_{3}}\right)$, whereas $\overline{\mathrm{C}}_{3}$ indicates a recognized positive constant.

\section{Enhanced SoSMC using SAR Optimization Algorithm}

\subsection{SMC Controller Modeling}

To indicate a lower order system the SMC modeling [28] contract with the sliding mode surface modeling, $\mathrm{x}=0$ while evaluating by means of the chosen plant. Hence, it is represented as $\mathrm{x}=\mathrm{e}+[\lambda \times \mathrm{e}(\mathrm{t})]$, whereas $\lambda>0$ is represented as a parameter of the design. Subsequently, the modeling of the appropriate controller is performed so, which the sliding mode surface is stressed to achieve by the surface regarding finite time.

In eq. (11), the SMC mathematical model is developed, whereas $U_{\text {eq }}(t)$ and $U_{x}(t)$ denotes the equal and discontinuous segment of SMC.

$$
\mathrm{u}(\mathrm{t})=\mathrm{U}_{\mathrm{x}}(\mathrm{t})+\mathrm{U}_{\mathrm{eq}}(\mathrm{t})
$$

On the basis of the eq. (11), the variable $\mathrm{U}_{\mathrm{x}}(\mathrm{t})$ is implemented, whereas $\mu^{(1)}$ and $\mu^{(2)}$ indicates the positive gain of the model and $q$ indicates the sliding surface of SMC. On the basis of the eq. (12), the 
variables $\mathrm{U}_{\mathrm{x}}(\mathrm{t})$ and $\mathrm{U}_{\text {eq }}(\mathrm{t})$ are hence attained. Likewise, eq. (13) can be attained regarding the weighting factor $w t$.

$$
\begin{aligned}
& \mathrm{U}_{\mathrm{x}}(\mathrm{t})=\mu^{(1)} \int_{0}^{\mathrm{t}} \operatorname{sign}(\mathrm{x}) \mathrm{dt}+\mu^{(2)}|\mathrm{x}|^{0.5} \operatorname{sign}(\mathrm{x}) \\
& \mathrm{U}_{\mathrm{eq}}(\mathrm{t})=\mathrm{x}+\mathrm{e}+\mathrm{wt}
\end{aligned}
$$

\subsection{Design of SoSMC}

Using both the discontinuous and continuous modes, a consistent model of SMC [28] is produced. At a low frequency of the system, the continuous mode indicates the operating form, at high system frequency, the discontinuous mode of this design denotes the operating form. The alteration in the desired, and the current system state, is therefore hypothetical which needs to the sliding surface $x$. In the system, the incidence of chattering creates instant indefinite alterations in the control signal and allows FoSMC to suggest the minimization in the calculation. In reality, it is attained regarding the function sign that is unapproachable using the SoSMC. From eq. (10), the proposed SoSMC design is shown in eq. (14), whereas $w t$ indicates the weight included regarding the SMC model.

Hence, the SMC system sliding surface is attained by eq. (15), whereas, $\left(\mu^{(1)}, \mu^{(2)}, \phi^{0}, \mathrm{Q}(\mathrm{m})\right)$ specifies achievement from a positive viewpoint.

$$
\begin{aligned}
& \mathrm{u}(\mathrm{t})=\mathrm{U}_{\text {eq }}+\mathrm{U}_{\mathrm{q}} \cdot \mathrm{wt} \\
& \mu^{(1)}>\frac{\phi^{0}}{\mathrm{~L}(\mathrm{~m})} \geq 0 \text { and }\left(\mu^{(2)}\right)^{2} \geq 4 \frac{\phi^{0}}{(\mathrm{~K}(\mathrm{~m}))^{2}} \frac{\mu^{(2)} \mathrm{L}(\mathrm{m})+\phi^{0}}{\mu^{(2)} \mathrm{Q}(\mathrm{m})+\phi^{0}}>0 \\
& \mathrm{x}=\lambda \mathrm{e}+\dot{\mathrm{e}}
\end{aligned}
$$

The investigational weight factor must be varied adaptively to attain the required control signal. Hence, when influencing the disparity among the required and the calculated angle, in the signal, the error must be minimized.

\subsection{Optimization of SoSMC}

The system of AUV creates tracking errore which is shown in Eq. (17). Moreover, the error must be reduced by means of the predetermined wt (weight factor). Hence, the performed SoSMC approach examines a weighting factor that is changed using the SRO algorithm [30].

The objective model of this algorithm aspires at reducing the concluding $e$ which is indicated as an error, as stated in Eq. (17).

$$
J=\min (e)
$$

\section{SAR Optimization Approach}

Here, the formulation algorithm of the proposed approach [30] in order to solve a maximization issue is explained. In the SAR algorithm, the locations of the humans' are equivalent to the solutions of the optimization issue, and the number of clues establish in these locations indicates the objective model for these solutions. Fig 3 demonstrates the flowchart of SAR.

\subsection{Clues}

During the search, the group members collect clue information. They left a few clues when they found enhanced clues in other locations, although information on them is exploited to get better operations in searching. In the proposed method, the left clues' location is saved in the matrix of memory (matrix P), while the humans' locations are saved in a matrix of location (matrix $\mathrm{Y}$ ). The matrix dimensions $\mathrm{P}$ are equivalent to those of the matrix $Y$. They are $\mathrm{N} \times \mathrm{D}_{\mathrm{m}}$ matrices, whereas $\mathrm{D}_{\mathrm{m}}$ indicates the problem dimension and $N$ indicates the count of humans. The matrix of clues (matrix $\mathrm{C}_{\mathrm{m}}$ ) indicates a matrix comprising the locations of established clues. This matrix comprises of 2 matrices $\mathrm{Y}$ and P. Eq. (18) indicates how to generate $\mathrm{C}_{\mathrm{m}}$. Each and every new solution in individual and social stages is generated on the basis of the clues matrix, and it is a significant element of SAR. In each human search stage, the matrices $\mathrm{Y}, \mathrm{P}$, and $\mathrm{C}_{\mathrm{m}}$ are updated: 


$$
\mathrm{C}_{\mathrm{m}}=\left[\begin{array}{l}
\mathrm{Y} \\
\mathrm{P}
\end{array}\right]=\left[\begin{array}{lll} 
& & \\
\mathrm{Y}_{11} & \cdots & \mathrm{Y}_{1 \mathrm{D}_{\mathrm{m}}} \\
\vdots & \ddots & \vdots \\
\mathrm{Y}_{\mathrm{N} 1} & \cdots & \mathrm{Y}_{\mathrm{ND}_{\mathrm{m}}} \\
\mathrm{P}_{11} & \cdots & \mathrm{P}_{1 D_{\mathrm{m}}} \\
\vdots & \ddots & \vdots \\
\mathrm{P}_{\mathrm{N} 1} & \cdots & \mathrm{P}_{\mathrm{ND}_{\mathrm{m}}}
\end{array}\right]
$$

In eq. (18), $\mathrm{P}$ and $\mathrm{Y}$ states memory and humans' location matrices, correspondingly, and $\mathrm{Y}_{\mathrm{N} 1}$ states the location of the $1^{\text {st }}$ dimension for the $\mathrm{N}^{\text {th }}$ human. In addition, $\mathrm{P}_{1 \mathrm{D}_{\mathrm{m}}}$ indicates the location of the $\mathrm{D}_{\mathrm{m}}^{\text {th }}$ dimension for the 1st memory. The 2 stages of human searches, and the individual and social phase, are formed as below.

\subsection{Social Stage}

Taking into consideration of the descriptions stated in the preceding section, and in view of a random clue between establishing clues, the search direction is attained by utilizing the eq. (19).

$$
\mathrm{SD}_{\mathrm{j}}=\left(\mathrm{Y}_{\mathrm{j}}-\mathrm{C}_{\mathrm{m}_{\mathrm{k}}}\right) ; \mathrm{k} \neq \mathrm{j}
$$

In eq. (19), $\mathrm{Y}_{\mathrm{j}}, \mathrm{C}_{\mathrm{mk}}$, and $\mathrm{SD}_{\mathrm{j}}$ are the location of the $\mathrm{j}^{\text {th }}$ human, the location of the $\mathrm{k}^{\text {th }}$ clue, and the direction of search for $j^{\text {th }}$ human, correspondingly. $k$ indicates a random integer number varies from one and two $N$ and selected in an approach which $\mathrm{k} \neq \mathrm{j}$.

It is significant to indicate which humans usually search to facilitate; each and everyone required regions, which are explored and some repeated position is not searched for a second time. Consequently, the search must be performed in a way that a group member's movement to each other is restricted. Finally, all dimensions $Y_{j}$ must not be altered by moving in the direction of eq. (19). In order to use this restraint, the binomial crossover operator is exploited. If consider clue is superior to the clue associated with the present location, a region around $\mathrm{SD}_{j}$ direction and approximately the location of that clue is searched; or else, the search will carry on approximately the present position beside the $\mathrm{SD}_{\mathrm{j}}$ direction. At last, the eq. (20) is exploited for the social phase:

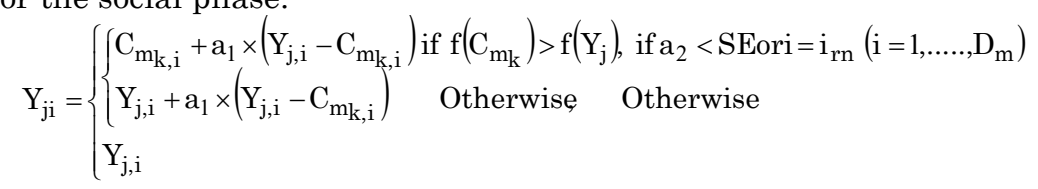

In eq. (20), $\mathrm{Y}_{\mathrm{j}, \mathrm{i}}^{\prime}$ denotes the novel location of $\mathrm{i}^{\text {th }}$ dimension for the $\mathrm{j}^{\text {th }}$ human; $\mathrm{C}_{\mathrm{m}_{\mathrm{k}, \mathrm{i}}}$ indicates location of the $i^{\text {th }}$ dimension for $k^{\text {th }}$ establish clue; $f\left(\mathrm{C}_{\mathrm{m}_{\mathrm{k}}}\right)$ and $\mathrm{f}\left(\mathrm{Y}_{\mathrm{j}}\right)$ indicates the values of the objective model for the solutions $\mathrm{C}_{\mathrm{m}_{\mathrm{k}}}$ and $\mathrm{Y}_{\mathrm{j}}$, correspondingly; $\mathrm{a}_{1}$ indicates a random count with a uniform distribution in assortment $[-1,1] ; a_{2}$ indicates a uniformly distributed random count in assortment $[0,1]$ and it is diverse for every dimension, however, $a_{1}$ is fixed for all dimensions; $i_{r n}$ indicates a random integer number ranging among one and $\mathrm{D}_{\mathrm{m}}$ that assures that as a minimum 1 dimension of $\mathrm{Y}_{\mathrm{j}, \mathrm{i}}^{\prime}$ is diverse from $\mathrm{Y}_{\mathrm{j}, \mathrm{i}}$; and $\mathrm{SE}$ is a algorithm parameter assortment among zero and one. Eq. (20) is exploited to attain an original location of the $\mathrm{j}^{\text {th }}$ human in each and every dimension.

\subsection{Individual Stage}

In this stage, in the region of human's present location they will search, and the initiative of linking diverse clues exploited in the social stage is used to explore. In opposition to the social stage, each and every dimension of $Y_{j}$ alter in the individual stage. The novel location of $j^{\text {th }}$ humans is attained using eq. (21).

$$
\mathrm{Y}_{\mathrm{j}}^{\prime}=\mathrm{Y}_{\mathrm{j}}+\mathrm{a}_{3} \times\left(\mathrm{C}_{\mathrm{m}_{\mathrm{k}}}-\mathrm{C}_{\mathrm{m}_{\mathrm{p}}}\right), \mathrm{j} \neq \mathrm{k} \neq \mathrm{p}
$$


In eq. (21), $\mathrm{k}$ and $\mathrm{p}$ are random integer numbers deviating among 1 and $2 N$. To avert movement beside with erstwhile clues, $k$ and $p$ are selected in that manner, $j \neq k \neq p . a_{3}$ indicates a random number with a uniform distribution assortment between 0 and 1 .

\subsection{Boundary Rule}

In each and every meta-heuristic algorithm, in the solution space, the entire solutions must be positioned, and if they are the permissible solution space, the solution must be adapted. Consequently, if the novel location of a human is used due to the solution space, the eq. (22) is exploited to adapt novel location:

$$
\mathrm{Y}_{\mathrm{ji}}^{\prime}=\left\{\begin{array}{l}
\frac{\left(\mathrm{Y}_{\mathrm{ji}}+\mathrm{Y}_{\mathrm{i}}^{\max }\right)}{2}, \text { if } \mathrm{Y}_{\mathrm{ji}}^{\prime}>\mathrm{Y}_{\mathrm{i}}^{\max }\left(\mathrm{i}=1, \ldots . ., \mathrm{D}_{\mathrm{m}}\right) \\
\frac{\left(\mathrm{Y}_{\mathrm{ji}}+\mathrm{Y}_{\mathrm{i}}^{\min }\right)}{2}, \text { if } \mathrm{Y}_{\mathrm{ji}}^{\prime}>\mathrm{Y}_{\mathrm{i}}^{\min }
\end{array}\right.
$$

In eq. (22), $\mathrm{Y}_{\mathrm{i}}^{\max }$ and $\mathrm{Y}_{\mathrm{i}}^{\min }$ indicates the utmost values, and the threshold, will be least for the $\mathrm{i}^{\text {th }}$ dimension, correspondingly.

\subsection{Updating Locations and Information}

The members in each group will look for consistency with these 2 stages, and subsequent to every stage In each iteration, if the objective model value in location $\mathrm{Y}_{\mathrm{j}}^{\prime}\left(\mathrm{f}\left(\mathrm{Y}_{\mathrm{j}}\right)\right)$ is higher than the preceding one $f\left(Y_{j}\right)$, the preceding location $\left(\mathrm{Y}_{\mathrm{j}}\right)$ will be saved in a random location of the memory matrix $(\mathrm{P})$ exploiting eq. (23) and this location will be established as a new location exploiting eq. (24). Or else, this location is missing and the memory is not restructured:

$$
\begin{aligned}
& P_{n}=\left\{\begin{array}{l}
Y_{j}, \text { if } f\left(Y_{j}^{\prime}\right)>f\left(Y_{j}\right) \\
P_{n}, \text { otherwise }
\end{array}\right. \\
& Y_{j}=\left\{\begin{array}{l}
Y_{j}^{\prime}, \text { if } f\left(Y_{j}^{\prime}\right)>f\left(Y_{j}\right) \\
Y_{j}, \text { otherwise }
\end{array}\right.
\end{aligned}
$$

In eq. (23), $P_{n}$ indicates the location of the $n^{\text {th }}$ saved clue in the memory matrix and $n$ is an random integer number ranging among 1 and $\mathrm{N}$. By exploiting this kind of updating of the memory raises a variety of technologies and the capability of the algorithm to discover the global best possible too.

\subsection{Discarding Clues}

In operations of SAR, time is considered a significant aspect as missing people might be wounded and the SAR teams delay might cause human deaths. Hence, aforesaid operations should be performed so that the main space is explored in the straight probable time. Therefore, if a human cannot observe, improved clues subsequent to a definite count of searches around her/his present location; she/he lefts from the present position and moves towards an original location. To form this behavior, initially, USN is set to 0 for every human being. When a human discovers enhanced clues in the primary else secondary stage of the search, the USN is considered as 0 for that human; or else, it will augment by one point as stated in eq. (25).

$$
\mathrm{USN}_{\mathrm{j}}=\left\{\begin{array}{l}
\mathrm{USN}_{\mathrm{j}}+1 . \mathrm{if}\left(\mathrm{Y}_{\mathrm{j}}^{\prime}\right)<\mathrm{f}\left(\mathrm{Y}_{\mathrm{j}}\right) \\
0, \quad \text { otherwise }
\end{array}\right.
$$

In eq. (25), $\mathrm{USN}_{\mathrm{j}}$ states the number of occurrences the human $\mathrm{j}$ is not capable to discover improved clues. For a human, while the USN is higher than the MU search number, she/he leaves to a random location in search space by exploiting eq. (26), which $U S N_{j}$ is set to 0 for that human:

$$
\mathrm{Y}_{\mathrm{ji}}=\mathrm{Y}_{\mathrm{i}}^{\min }+\mathrm{a}_{4} \times\left(\mathrm{Y}_{\mathrm{i}}^{\max }-\mathrm{Y}_{\mathrm{i}}^{\min }\right), \mathrm{i}=1, \ldots, \mathrm{D}_{\mathrm{m}}
$$

In eq. (26), $a_{4}$ indicates an random count with a uniform distribution which ranges among zero and one. It is diverse for every dimension.

\subsection{SAR Parameters control}

SAR contains 2 control parameters: MU and SE and search number. In the social stage, the SE is exploited to manage the result of group members on every other. This parameter varies in $[0,1]$. Higher 
SE values raise the rate of the convergence and besides reduce the global search capability of the algorithms. The parameter of MU shows the utmost number of ineffective searches ahead of leaving a clue. It ranges within $\left[0,2 \times \mathrm{T}_{\max }\right]$, whereas $2 \times \mathrm{T}_{\max }$ is the utmost count of searches performed by every human and $\mathrm{T}_{\max }$ indicates the utmost count of iterations. For advanced MU values, humans will certainly not go away from the clues. Conversely, the least parameter values guide to the group three members come to an end searching in the region of the present clue and leave to other positions ahead of she/he can totally search around it. In contrast, high values of this parameter reason a rise in searches in the region of one clue and a decrease in the likelihood of searching in previous areas. The MU in a straight line associated with the dimension of the issue. As the search space rises, the utmost count of ineffective searches is improved, also.

For all the subsequent experimentations, the value of $\mathrm{SE}$ was set to 0.05 and the MU value was attained using equation (27). The evaluation of SAR parameters encompasses exhibited that these SE and MU values are appropriate in order to solve the single-objective continuous optimization issues.

$$
\mathrm{MU}=70 \times \mathrm{D}_{\mathrm{m}}
$$

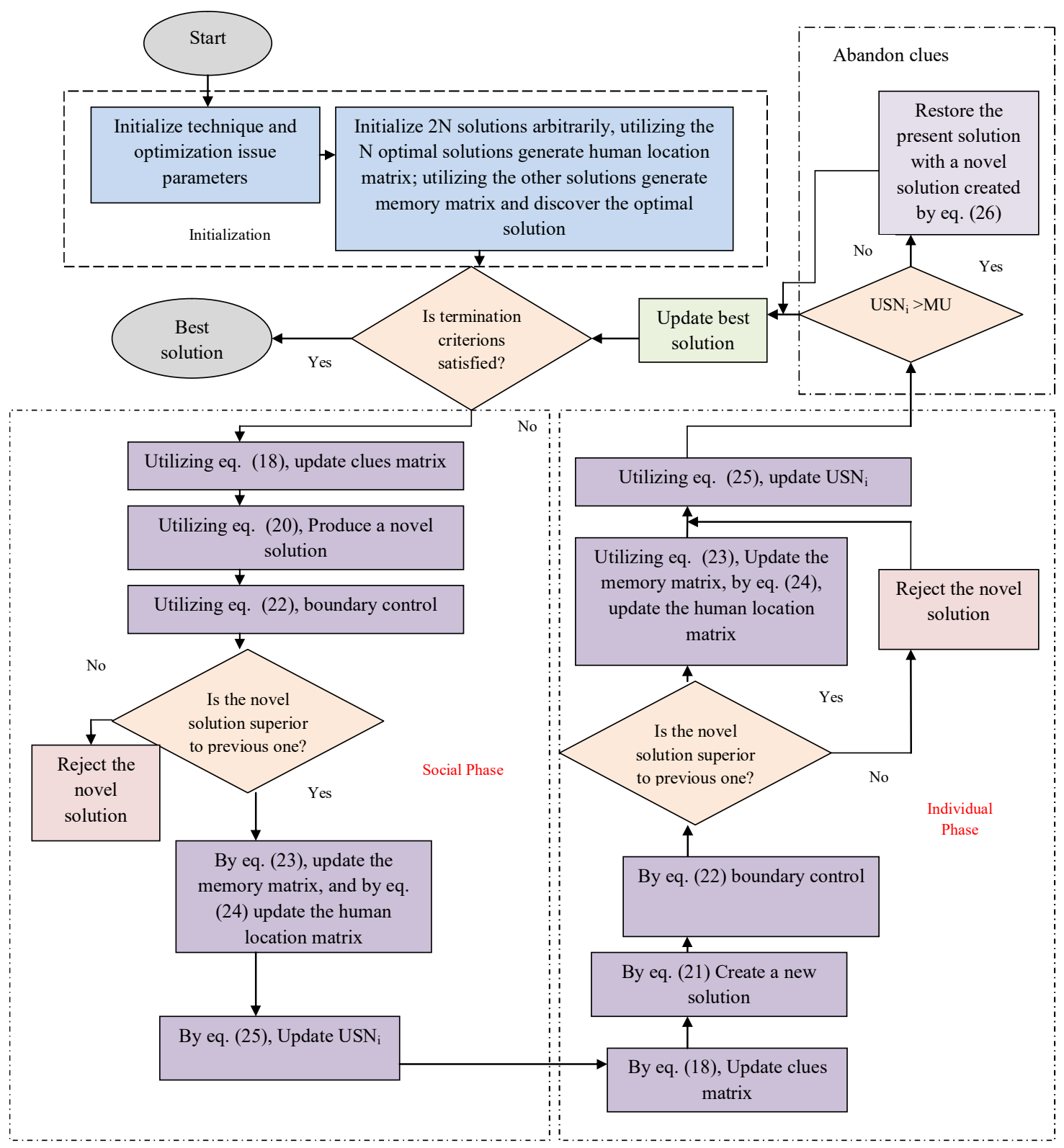

Fig. 3. Flow chart of the proposed SAR optimization algorithm

\section{Results and Discussions}




\subsection{Simulation Procedure}

In this section, the proposed algorithm is used in AUV in order to control the yaw angle was applied in MATLAB, and outcomes were examined. Moreover, the proposed algorithm was compared with the conventional SoSMC algorithms such as GA-SoSMC, FF-SoSMC, GW-SMC, ABC- SoSMC and GSOSoSMC algorithms and the experimentation outcomes were attained.

\subsection{Performance Evaluation}

Here, the evaluation of RMSE in order to control the yaw angle in the AUV system in various step responses is shown in Fig 4. In Fig 4, the set points such as set points 1, 2, 3, and 4. The analysis shows the proposed approach is analyzed with the traditional algorithms. In Fig 4, the proposed algorithm is $22 \%$ better than the GA-SoSMC, 31\% better than the FF-SoSMC, 15\% better than the GW-SMC, $32 \%$ better than the ABC-SoSMC and 21\% better than the GSO-SoSMC algorithms. Hence, the overall analysis shows the performance of the proposed approach exhibits better performance than the traditional algorithms.

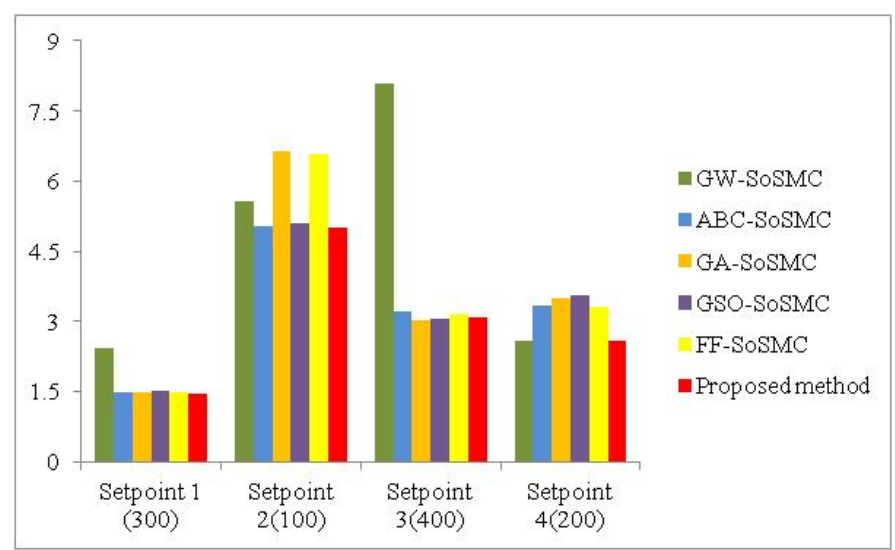

Fig. 4. Graphical representation of the proposed model with respect to the RMSE analysis

\section{Conclusion}

In defense organizations, AUV discovers widespread applications for submerged mine recognition and area examination. Additionally, it is helpful for gas and oil industries in recognition of leakage which is happened in the pipelines and in addition to numerous erstwhile marine industries. Moreover, confronts facade by AUV needed a satisfactory technical response to increase a well-organized controller which attains the problems of the maximum level security system. Therefore, this work has presented an approach in AUV, which is called SR-SoSMC in order to control the yaw angle. The proposed algorithm possesses minimize errors that are between desired yaw angles and predicted yaw angles for improving the system performance. The efficiency of the proposed SR-SoSMC method was shown by evaluating it with several other conventional algorithms. From the evaluation outcomes, it was obvious that the efficiency of the proposed algorithm in AUV is used in order to control the yaw angle with reduced error was attained.

\section{Compliance with Ethical Standards}

Conflicts of interest: Authors declared that they have no conflict of interest.

Human participants: The conducted research follows the ethical standards and the authors ensured that they have not conducted any studies with human participants or animals.

\section{References}

[1] Z. Yan, B. Song, Y. Zhang, K. Zhang, Z. Mao and Y. Hu, "A Rotation-Free Wireless Power Transfer System With Stable Output Power and Efficiency for Autonomous Underwater Vehicles," IEEE Transactions on Power Electronics, Volume. 34, Issue. 5, Page number. 4005-4008, May 2019. 
[2] B. Sun, D. Zhu, C. Tian and C. Luo, "Complete Coverage Autonomous Underwater Vehicles Path Planning Based on Glasius Bio-Inspired Neural Network Algorithm for Discrete and Centralized Programming," IEEE Transactions on Cognitive and Developmental Systems, Volume. 11, Issue. 1, Page number. 73-84, March 2019.

[3] B. Zhang, Y. Wang, H. Wang, X. Guan and Z. Zhuang, "Tracking a Duty-Cycled Autonomous Underwater Vehicle by Underwater Wireless Sensor Networks," IEEE Access, Volume. 5, page number. 18016-18032, 2017.

[4] M. Shinohara et al., "Development of a High-Resolution Underwater Gravity Measurement System Installed on an Autonomous Underwater Vehicle," IEEE Geoscience and Remote Sensing Letters, Volume. 15, Issue no. 12, page number. 1937-1941, December. 2018.

[5] Y. Li, Y. Wang, W. Yu and X. Guan, "Multiple Autonomous Underwater Vehicle Cooperative Localization in Anchor-Free Environments," IEEE Journal of Oceanic Engineering, Volume. 44, Issue no. 4, page number. 895911, October. 2019.

[6] W. A. Pineda MuñOz, A. Gauthier Sellier and S. GomàRiz Castro, "The Predictive Functional Control and the Management of Constraints in GUANAY II Autonomous Underwater Vehicle Actuators," IEEE Access, Volume. 6, page number. 22353-22367, 2018.

[7] A. Li, L. Ye, J. Yanqing, L. Yueming, C. Jian and H. Jiayu, "Soft-Switching Proximate Time Optimal Heading Control for Underactuated Autonomous Underwater Vehicle," IEEE Access, Volume. 7, page number. 143233143249, 2019.

[8] YoergerD, Slotine J," Robust trajectory control of underwater vehicles”, IEEE J Ocean Eng, Volume 10, issue number 4, page number. 462-470, 1985.

[9] Arimoto S, Miyazaki F," Stability and robustness of PID feedback control for robot manipulators of sensor capability”, In:Brady M, Paul R (eds) Robot research. MIT Press, Cambridge, page number. 783-799,1984.

[10] Goheen KR, Jefferys ER,” Multivariable self-turning autopilots for autonomous underwater vehicles”, IEEE J Ocean Eng, Volume 15, Issue number: 3, page number 144-151, 1990.

[11] Nakamura Y, Savant S,"Nonlinear tracking control of autonomous underwater vehicles", In: Proceedings of IEEE international conference on robotics and automation, Volume 3, page number: 44-49, 1992.

[12] Antonelli G, Chiaverini S, Sarkar N,WestM," Adaptive control of an autonomous underwater vehicle: experimental results on ODIN", IEEE Trans Control Syst Technol, Volume 9, Issue number: 5, page number: 756-765, 2001.

[13] Pisano A, Usai E,'Output-feedback control of an underwater vehicle prototype by higher-order sliding modes”, Automatica Volume 40, page number: 1525-1531, 2004.

[14] Silvestre C, Pascoal A, Kaminer I, "On the design of gain scheduled trajectory tracking controllers", Int J Robust Nonlinear Control, Volume 12, page number:797-839, 2002.

[15] Triantafyllou MS, Grosenbaugh MA,"Robust control for underwater vehicle systems with time delays”, IEEE J Ocean Eng, Volume 16, Issue number 1, page number:146-151, 1991.

[16] Feng Z, Allen R,"Reduced order Hœ control of an autonomous underwater vehicle.”, Control Eng Pract, Volume 13, page number:1511-1520, 2004.

[17] DeBitetto PA," Fuzzy logic for depth control of unmanned undersea vehicles”, IEEE J Ocean Eng, Volume 20, Issue number 3, page number 242-248, 1995.

[18] Yuh J," A neural net controller for underwater robotic vehicles”,IEEE J Ocean Eng, Volume 15, Issue number 3, page number 161-166, 1990.

[19] Moura A, Rijo R, Silva P, Crespo S () A multi-objective genetic algorithm applied to autonomous underwater vehicles for sewage outfall plume dispersion observations. Appl Soft Comput, Volume 10, Issue number 4, page number 1119-1126, 2010.

[20] Lee PM, Hong SW, Lim YK, Lee CM, Jeon BH, Park JW (1999) Discrete-time quasi-sliding mode control of an autonomous underwater vehicle. IEEE J Ocean Eng, Volume 24, Issue number 3, page number 388-395, 1999.

[21] T. I. Fossen, Guidance and control of ocean vehicles, John Wiley and Sons, Inc, 1994.

[22] Y. Shtessel, C. Edwards, L. Fridman, A. Levant, Sliding mode control and observation, Volume 10, Springer, 2014.

[23] Y. Shtessel, M. Taleb, F. Plestan, "A novel adaptive-gain supertwisting sliding mode controller: Methodology and application”, Automatica, Page number 759-769, 2012.

[24] T. Gonzalez, J. A. Moreno, L. Fridman, "Variable gain super-twisting sliding mode control", IEEE Transactions on Automatic Control, volume: 57, page number 2100-2105, 2012.

[25] N. Q. Hoang, E. Kreuzer, "A robust adaptive sliding mode controller for remotely operated vehicles", Technische Mechanik, volume 28, page number: 185-193, 2008.

[26] R. Cui, X. Zhang, D. Cui, "Adaptive sliding-mode attitude control for autonomous underwater vehicles with input nonlinearities", Ocean Engineering, volume 123 ,page number 45-54, 2016.

[27] Rongxin Cui, Xin Zhang and Dong Cui, "Adaptive sliding-mode attitude control for autonomous underwater vehicles with input nonlinearities," Ocean engineering, volume.123, page number. 45-54, September 2016.

[28] D. Ribas, P. Ridao, J. Neira, and J. D. Tardos, "SLAM using an imaging sonar for partially structured underwater environments," Proc. IEEE/RSJ Int. Conf. Intell. Robots Syst., Beijing, China, page number.. 50405045, October 2006.

[29] Galeani,Sergio, Tarbouriech,Sophie, Turner,Matthew, Zaccarian,Luca, "A tutorial on modern anti-windup design”, .Eur.J.Control, volume. 15, Issue. 3, page number. 418-440, 2009.

[30] Amir Shabani,Behrouz Asgarian, Saeed Asil Gharebaghi, Miguel A. Salido, and Adriana Giret,"A New Optimization Algorithm Based on Search and Rescue Operations",Mathematical Problems in Engineering Volume: 23 , 2019. 